Original Article

\title{
Electromyographic analysis of an ergonomic risk factor: overhead work
}

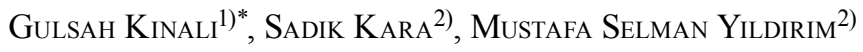 \\ 1) Physiotherapy and Rehabilitation Department, Istanbul Gelişim University College of Health: 34310 , \\ Avcllar, Istanbul, Turkey \\ 2) Institute of Biomedical Engineering, Fatih University, Turkey
}

\begin{abstract}
Purpose] Repetitive upper extremity exercises result in overuse injuries. However, it is challenging to identify the specific causative movements. This study evaluated the effects of different shoulder positions on grip and electrophysiological activity of upper extremity muscles. [Subjects and Methods] Forty subjects ranging from 18 and 30 years of age were analyzed. Surface electromyography and hand grip strength were measured during a range of shoulder exercises and numerous signal processing methods were applied. [Results] The maximum electromyographic activity intensity was observed in the wrist extensors at various angles. Deltoid activity rose significantly during shoulder flexion. [Conclusion] Overhead work causes shoulder muscle fatigue and prevents effective hand function, which affects occupational health and efficiency. Overhead work involves a hazardous position and decreases efficiency. Ergonomic solutions should be developed to prevent muscle fatigue and decreased grip force. Key words: Ergonomics, Work-related musculoskeletal disorders, Electromyography
\end{abstract}

(This article was submitted Jan. 19, 2016, and was accepted Mar. 13, 2016)

\section{INTRODUCTION}

Repetitive upper extremity exercises result in overuse injuries; however, it is challenging to identify the causative exercises. Additionally, the causes of one-third of occupational disorders are not classified. Therefore, the application of evidence-based healing and prevention is more complicated ${ }^{1}$. The upper extremities are primarily at risk of trauma and overuse injuries due to their functional importance. High levels of static contraction and prolonged static loads, as well as inappropriate posture, may lead to upper extremity disorders. Work-related disorders are defined as injuries of the muscles, joints, and ligaments caused by work activities. Increased absence due to sickness in recent years has been a trouble making issue in industrial society such as automotive production and poultry ${ }^{2,3)}$.

Working above shoulder level is commonly believed to lead to upper extremity disorders. Prolonged shoulder elevation causes shoulder muscle fatigue, which may lead to disorders ${ }^{4-6)}$.

The upper extremities are characterized by their motility, grip, and maneuverability, and are a very mobile and dynamic part of the body. Each upper extremity is comprised of the shoulder complex, arm, forearm, and hand. The close association of these components makes analyzing a single function difficult. The primary function of the shoulder complex is to position the upper extremity for functional utilization of the arm and hand, allowing the hand to perform its necessary gripping duty. Therefore, shoulder movements and grip are biomechanically associated ${ }^{6}$.

Grip is necessary for successful completion of daily activities. Grip force measurement is a regional evaluation method, and has been a criterion for hand function evaluation. It has recently been the preferred functional test to determine functional capacity and to evaluate work-related disorders and prevention methods ${ }^{7}$.

Although previous studies have assessed the risks of exercising above shoulder level, to our knowledge, no studies have simultaneously monitored the electromyographic (EMG) activity of the muscles and grip. The main objective of this study

\footnotetext{
*Corresponding author. Gulsah Kinali (E-mail: gulsah.kinali@gmail.com)

C2016 The Society of Physical Therapy Science. Published by IPEC Inc.

This is an open-access article distributed under the terms of the Creative Commons Attribution Non-Commercial No Derivatives (by-nc-nd) License $<$ http://creativecommons.org/licenses/by-nc-nd/4.0/>.
} 
was to investigate the electrophysiological outcomes of exercising above shoulder level, a major risk factor for work-related upper extremity disorders, and its influence on grip in different working angles of the arm.

\section{SUBJECTS AND METHODS}

Forty healthy Gelişim University College of Health Sciences students between the ages of 18 and 31 years participated in the study (20 females and 20 males). The average ages of the female and male subjects were $21.6 \pm 4.1$ and $22 \pm 2.6$ years, respectively.

Their average body mass index was $22.01 \pm 3.4$ and $24 \pm 4.4 \mathrm{~kg} / \mathrm{m}^{2}$, respectively.

Recordings with extreme noise; where the participant misunderstood the directions; or with short grip duration were not considered to increase accuracy and were not included in the study. Students with neurological disability due to musculoskeletal disease, severe cardiopulmonary disease, severe hearing loss, history of surgery within the past six months, history of epilepsy, or chronic neurologic disease were not included.

Before the study, documentation of medical ethics was obtained from the local ethics committee of İstanbul Sadi Konuk Education and Research Hospital (decision no 2015/06), and the project received approval from the Gelisim University Administration; each participant also provided written informed consent.

EMG signals and hand grip strength were recorded using a BIOPAC system, a data acquisition hardware developed for educational and research purposes that acquires physiological signals, especially electrophysiological signals such as electromyogram, electroencephalogram, and electrocardiogram. Upon arrival, the students were asked to rest for $30 \mathrm{~min}$ and refrain from overexerting their hands. Students with hairy arms were requested to get a trim. Participants were informed on the objectives, measurement procedures, grip duration, rest breaks, movement positions, and repetitions. For the measurements, electrodes $(2-\mathrm{cm}$ diameter $\mathrm{Ag}-\mathrm{Ag} / \mathrm{Cl})$ were placed at $0.5-\mathrm{cm}$ intervals on the anterior deltoid and motor points of the wrist extensors and flexors, as determined by a physiotherapist. Grounding electrodes were placed on the ulnar styloid, olecranon, and forehead. Grip strength was measured in pounds, and EMG measurements were between 0 and 1,000 MHz. Measurements were taken in five positions with three repetitions, including four shoulder angles and a standard grip ${ }^{8-10,13,14)}$.

Surface electrodes were placed on the anterior deltoid and the hand wrist flexors and extensors. The motor points were identified on each participant by a physiotherapist who palpated the isometric contraction at the middle of the joint. A pivot was used to mark the grip and flexion points at $0^{\circ}, 45^{\circ}, 90^{\circ}$, and $135^{\circ}$ (Table 1 ); for each participant, a physiotherapist marked the points with removable colored dough. Motor point electrodes were placed on each muscle at 0.5 -cm intervals. Grounding electrodes were placed on the olecranon for flexors, the ulnar styloid for extensors, and the forehead for the deltoid. Channels were connected in the following order throughout the study: 1) hand dynamometer, 2) extensors, 3) flexors, and 4) deltoid. Measurements were taken at $0^{\circ}, 45^{\circ}, 90^{\circ}, 135^{\circ}$, and standard grip, respectively, for three repetitions per position. A 30 -s break was given between each measurement. Directions were given based on the chronometer. The reference point was $0^{\circ}$ with the arm at rest near the trunk and no shoulder movement ${ }^{12,14,15)}$.

Flexion and extension were measured with a goniometer, and colored markers were placed at $45^{\circ}$ (yellow), $90^{\circ}$ (orange), and $135^{\circ}$ (red).

Electromyogram signals and hand grip measurements were recorded using a BIOPAC system (Biopac Systems, Santa Barbara, CA, USA). Measurements from individual participants were saved as a single file and the recordings lasted for $8.5 \mathrm{~min}$. The resultant files were analyzed using MATLAB, a computational tool and programming language used in academia, engineering, science, economics, and industry. During the procedure, 15 gripping actions were performed, and an algorithm was optimized to calculate the instant of gripping. Inside the signal of gripping force, the points greater and lower the mean value are determined, and the points greater than the mean value are defined as gripping point. In order to increase the accuracy of the algorithm, the gaps in the gripping signal that are shorter than 3 seconds are connected, and gripping instants shorter than $3 \mathrm{~s}$ were eliminated. Root mean square (RMS) values of EMG signals, corresponding to gripping instants, were calculated. The average values of 3 repeated gripping actions were calculated; the entire process was repeated for each participant and the average value for the group was calculated. Thus, the results of three different channels in five different positions for each participant were compared based on their RMS values.

Table 1. Shoulder flexion degrees and position names

\begin{tabular}{lc}
\hline Shoulder flexion & Position name \\
\hline $0^{\circ}$ & 1 \\
$45^{\circ}$ & 2 \\
$90^{\circ}$ & 3 \\
$135^{\circ}$ & 4 \\
$0^{\circ}$ Shoulder flexion, $90^{\circ}$ Elbow flexion (Standard position) & 5 \\
\hline
\end{tabular}




\section{RESULTS}

The maximum grip time for each subject was estimated using the force measuring signal. The RMS of the related values were calculated. First, the average of the repetitions was calculated for every participant, before calculating the group mean. Table 2 shows that the greatest activity in grip occurred in the extensors $(\mathrm{p}<0.05)$. As the angle of shoulder flexion increased, the deltoid activity increased at twice the rate (Table 3$)(\mathrm{p}<0.05)$. The most active muscles during grip and shoulder flexion were the wrist extensors; however, as shoulder flexion increased, the deltoid muscle was activated. Activity in the hand wrist flexors did not change with shoulder flexion (Table 4). As shoulder flexion increased, the overall muscle activity increased while grip force decreased (Table 5).

\section{DISCUSSION}

Previous studies have shown overhead exercises to be a risk factor for upper extremity disorders. This study focused on changes in upper extremity EMG values and grip force at different degrees of shoulder flexion. The objective was to assess the effect of overhead work on the electrophysiology of upper extremity muscles and hand function ${ }^{1,4)}$.

The results of the current study showed that working above shoulder level was the most exhausting position for upper extremity muscles. Hand function deteriorated with increasing arm angle. Thus, overhead work is a risk factor for work-related upper extremity disorders, and grip force deteriorates as the muscles fatigue while stabilizing the arm in an upright position. Finally, in addition to reach and grip, the upper extremity muscles also play a crucial role in stabilization.

Won-Gyu Yoo et al. investigated the effect of exercises above shoulder level on electrophysiological muscular activity. They found that overhead exercises cause fatigue in the superior trapezius and discomfort in the shoulder joint by affecting the scapular position. The results of the current study revealed that the deltoid was highly activated at $135^{\circ}$ shoulder flexion compared to other shoulder muscles. Based on these findings, overhead work causes fatigue in muscles with direct and indirect shoulder insertions ${ }^{4}$.

Hagg et al. reported that wrist flexors showed more fatigue than extensors during grip in their electrophysiological research on grip force ${ }^{16}$. The results of the current study also showed that wrist extensors were more active. Thus, extensors were crucial for stabilizing the upper extremities. Although the flexors performed finger flexion for the initial part of grip, the wrist extensors became more active in order to stabilize the wrist. The results of the current study suggest that the upper extremities exert considerable energy for stabilization in addition to its more common functions of reaching and gripping. Grip force is closely related to upper extremity force. The shoulder joint provides stabilization during grip. The main function of the shoulder is flexion, and it is frequently used in daily activities. Therefore, the current study investigated how shoulder flexion influences grip force. Research on this relationship is scarce ${ }^{7,11,15)}$. In our research, the greatest grip force was observed in

Table 2. Extensor muscle EMG results $(\mathrm{N}=40)$

\begin{tabular}{cc}
\hline Position & Mean \pm SD \\
\hline $1^{*}$ & $0.39 \pm 0.18$ \\
2 & $0.35 \pm 0.17$ \\
3 & $0.35 \pm 0.16$ \\
4 & $0.34 \pm 0.16$ \\
5 & $0.32 \pm 0.17$ \\
\hline
\end{tabular}

$* \mathrm{p}<0.05$, each position was compared to the fourth position.

Table 4. Flexor muscle EMG results $(\mathrm{N}=40)$

\begin{tabular}{cl}
\hline Position no & Mean \pm SD \\
\hline 1 & $0.19 \pm 0.10$ \\
2 & $0.20 \pm 0.09$ \\
3 & $0.21 \pm 0.08$ \\
4 & $0.21 \pm 0.08$ \\
5 & $0.20 \pm 0.10$ \\
\hline
\end{tabular}

Table 3. Deltoid muscle EMG results $(\mathrm{N}=40)$

\begin{tabular}{cl}
\hline Position no & Mean \pm SD \\
\hline $1^{*}$ & $0.16 \pm 0.71$ \\
2 & $0.25 \pm 0.65$ \\
3 & $0.28 \pm 0.56$ \\
4 & $0.32 \pm 0.49$ \\
$5^{*}$ & $0.15 \pm 0.46$ \\
\hline
\end{tabular}

$* \mathrm{p}<0.05$, each position was compared to the fourth position.

Table 5. Grip force results $(\mathrm{N}=40)$

\begin{tabular}{cc}
\hline Position no & Mean \pm SD \\
\hline 1 & $32.14 \pm 14.98$ \\
2 & $29.56 \pm 12.84$ \\
3 & $29.37 \pm 13.48$ \\
4 & $28.90 \pm 13.19$ \\
5 & $26.94 \pm 13.12$ \\
\hline
\end{tabular}


$0^{\circ}$ shoulder flexion, which showed a reasonable decline with increasing shoulder flexion.

The findings of the current study indicate that the upper extremities exert force to lift and stabilize the arm during shoulder flexion, resulting in reduced grip force. Reduced grip force with increased shoulder flexion can be interpreted as hand function decline. Thus, overhead work causes shoulder muscle fatigue and prevents effective hand function, which affects occupational health and efficiency.

Overhead work is a crucial risk factor for work-related disorders. Because workers are unable to maintain adequate grip function, they become fatigued, which decreases work efficiency. Decreasing the force required for workers to hold their arms up will reduce the risk for disorders and increase efficient hand utilization, which improve worker health, efficiency, and workforce ${ }^{1,16)}$.

The results of the current study indicate that overhead work places workers in a hazardous position and decreases efficiency. Ergonomic solutions should be developed to prevent muscle fatigue and decreased grip force. For example, a carrying system might be used for weight carried by workers. Moreover, we recommend rotating tasks among overhead workers in order to protect the upper extremity muscles ${ }^{17,18)}$.

\section{REFERENCES}

1) Shin SJ, Yoo WG: Effects of overhead work involving different heights and distances on neck and shoulder muscle activity. Work, 2015, 51: 321-326. [Medline] [CrossRef]

2) Dokuztug F, Acik E, Aydemir A, et al.: Early symptoms of the work-related musculoskeletal disorders in hand and upper extremity in the poultry industry. J Med Sci, 2006, 6: 305-313. [CrossRef]

3) Kinali G: The correlations between physical fitness level of worker and work related physical risk factors and work related musculo skeletal disorder, A MSc. Thesis, 2008, pp 21-22.

4) Yoo WG: Comparison of the cervical extension angle and the upper trapezius muscle activity between overhead work and below-knee work. J Phys Ther Sci, 2013, 25: 1289-1290. [Medline] [CrossRef]

5) Shin SJ, An DH, Oh JS, et al.: Changes in pressure pain in the upper trapezius muscle, cervical range of motion, and the cervical flexion-relaxation ratio after overhead work. Ind Health, 2012, 50: 509-515. [Medline] [CrossRef]

6) Yoo WG: Changes in acromion and scapular position after short-term overhead work. J Phys Ther Sci, 2013, 25: 679-680. [Medline] [CrossRef]

7) Matheson LN, Isernhagen SJ, Hart DL: Relationships among lifting ability, grip force, and return to work. Phys Ther, 2002, 82: 249-256. [Medline]

8) Chau N, Pétry D, Bourgkard E, et al.: Comparison between estimates of hand volume and hand strengths with sex and age with and without anthropometric data in healthy working people. Eur J Epidemiol, 1997, 13: 309-316. [Medline] [CrossRef]

9) Mathiowetz V: Comparison of Rolyan and Jamar dynamometers for measuring grip strength. Occup Ther Int, 2002, 9: 201-209. [Medline] [CrossRef]

10) Shechtman O, Gestewitz L, Kimble C: Reliability and validity of the DynEx dynamometer. J Hand Ther, 2005, 18: 339-347. [Medline] [CrossRef]

11) Alley DE, Shardell MD, Peters KW, et al.: Grip strength cutpoints for the identification of clinically relevant weakness. J Gerontol A Biol Sci Med Sci, 2014, 69: 559-566. [Medline] [CrossRef]

12) De Luca CJ: The use of surface electromyography in biomechanics. J Appl Biomech, 1997, 13: 135-163.

13) Konrad P: The ABC of EMG, A Practical Introduction to Kinesiological Electromyography. 2006, 1.4.

14) Cho SH, Lee JH: Comparison of the amplitudes of the h-reflex of post-stroke hemiplegia patients and normal adults during walking. J Phys Ther Sci, 2013, 25: 729-732. [Medline] [CrossRef]

15) Hägg GM, Milerad E: Forearm extensor and flexor muscle exertion during simulated gripping work—an electromyographic study. Clin Biomech (Bristol, Avon), 1997, 12: 39-43. [Medline] [CrossRef]

16) Michael S, Walter K: Work-related musculoskeletal disorders in the automotive industry due to repetitive work-implications for rehabilitation. J Occup Med Toxicol, 2010, 5: 6 .

17) Viester L, Verhagen EA, Proper KI, et al.: VIP in construction: systematic development and evaluation of a multifaceted health programme aiming to improve physical activity levels and dietary patterns among construction workers. BMC Public Health, 2012, 12: 89. [Medline] [CrossRef]

18) Somnath G, Samrat D: Design and evaluation of ergonomic interventions for the prevention of musculoskeletal disorders in India. Ann Occup Environ Med, 2014, 26: 1 . 